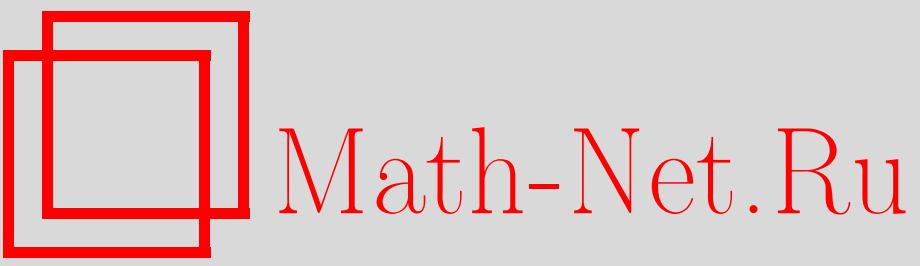

О. Н. Бирюков, Кручения на плоских диаграммах узлов, Итоги науки и техн. Сер. Соврем. мат. и ее прил. Темат. обз., 2021, том 194, 71-77

DOI: https://doi.org/10.36535/0233-6723-2021-194-71-77

Использование Общероссийского математического портала Math-Net.Ru подразумевает, что вы прочитали и согласны с пользовательским соглашением

http://www.mathnet.ru/rus/agreement

Параметры загрузки:

IP : 54.198 .67 .100

26 апреля 2023 г., 13:47:24 


\title{
КРУЧЕНИЯ НА ПЛОСКИХ ДИАГРАММАХ УЗЛОВ
}

\author{
(c) 2021 г. $\quad$ О. Н. БИРЮКОВ
}

\begin{abstract}
АннотАция. Рассматривается задача распознавания узлов как гладких вложений окружности в $\mathbb{R}^{3}$, заданных своими плоскими диаграммами. Вводится понятие кручения на плоской диаграмме и предлагается способ кодирования кручений и самих узлов с помощью Т-графов. Показывается, что подобный подход позволяет в некоторых ситуациях легко распознать тривиальный узел.
\end{abstract}

Ключевые слова: узел, распознавание тривиального узла, кручение, Т-граф.

\section{TWISTS ON FLAT KNOT DIAGRAMS}

\section{(c) 2021 O. N. BIRYUKOV}

\begin{abstract}
We consider the problem of recognizing knots as smooth embeddings of a circle into $\mathbb{R}^{3}$ given by their planar diagrams. The concept of a twist on a planar diagram is introduced and a method for encoding twists and nodes by T-graphs is proposed. We show that in some situations this approach allows one to recognize a trivial knot.
\end{abstract}

Keywords and phrases: knot, recognition of a trivial knot, twist, T-graph.

AMS Subject Classification: 57M25, 57M27

1. Введение. В работе предлагается новый подход к хорошо известной задаче распознавания узлов. Под узлом понимается гладкое вложение окружности $\mathbb{S}^{1}$ в $\mathbb{R}^{3}$, а также образ этого вложения. Два узла называются равными, если существует изотопный тождественному диффеоморфизм пространства $\mathbb{R}^{3}$, переводящий один узел в другой. Узел можно задать плоской диаграммой - проекцией узла на некоторую плоскость, имеющей только двойные точки самопересечения (перекрестки), причем в каждом перекрестке указана структура «проход-переход», т.е. указано, какая ветвь расположена в пространстве «выше» (дальше от плоскости проецирования), а какая «ниже». Задача распознавания узлов заключается в том, чтобы по двум заданным плоским диаграммам распознать, совпадают ли определяемые ими узлы. Обзор основных подходов к решению этой задачи можно найти в [1].

В данной работе предлагается новый комбинаторный подход к кодированию и распознаванию узлов. Вводится понятие кручения на плоской диаграмме узла и способ кодирования кручений с помощью графов с некоторой дополнительной структурой, называемых здесь Т-графами. Показывается, что Т-графы не только позволяют закодировать структуру кручений на плоской диаграмме, но и сам узел можно однозначно восстановить по Т-графу. Приводится пример плоской диаграммы тривиального узла, которую нельзя монотонно упростить движениями Рейдемейстера, а соответствующий Т-граф монотонно упрощается, что позволяет распознать тривиальность узла. 
2. Понятие кручения. Пусть задан узел $K$ и $D_{K}$-его плоская диаграмма. Ориентируем диаграмму $D_{K}$ и определим знак каждого перекрестка следующим образом:
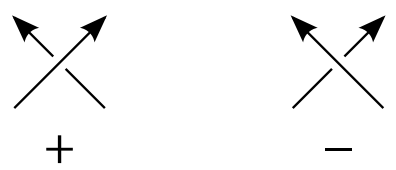

Плоскую диаграмму $D_{K}$ будем также рассматривать как планарный 4-валентный граф, вершины которого соответствуют перекресткам исходной диаграммы, и в каждой вершине ребра пересекаются под прямым углом. Поэтому перекрестки на диаграмме $D_{K}$ будем также называть ее вершинами.

Кручением на плоской диаграмме узла называется всякий двуугольник, стороны которого не пересекаются, а вершины, возможно, совпадают. Вершины этого двуугольника будем называть вершинами кручения, и также говорить, что между этими двумя вершинами (перекрестками на плоской диаграмме) имеется кручение. Стороны этого двуугольника пересекаются в его вершинах под прямым углом, каждый из этих углов будем называть направлением кручения в соответствующей вершине.

Кручение с вершинами $A$ и $B$ будем обозначать $A B$. Стороны кручения $A B$ обозначим $\alpha_{A B}$ и $\beta_{A B}$ так, чтобы в вершине $A$ кратчайший поворот от стороны $\alpha_{A B}$ к стороне $\beta_{A B}$ происходил против часовой стрелки. Аналогично, для сторон кручения $A B$ будем использовать обозначения $\alpha_{B A}$ и $\beta_{B A}$ в зависимости от их расположения в вершине $B$.

Пример 1. На следующей плоской диаграмме между вершиной $A_{1}$ и каждой из вершин $B_{1}$, $B_{2}, B_{3}$ есть кручение. Направления этих кручений выделены на рисунке дугами. Расставлены также знаки всех перекрестков, для чего выбрана ориентация. В каждой вершине плоской диаграммы есть четыре возможных направления кручений. Два из них, выделенные дугами на рисунке и зависящие от того, как соединяются четыре конца диаграммы, будем называть циклическими, а два другие - остовными.
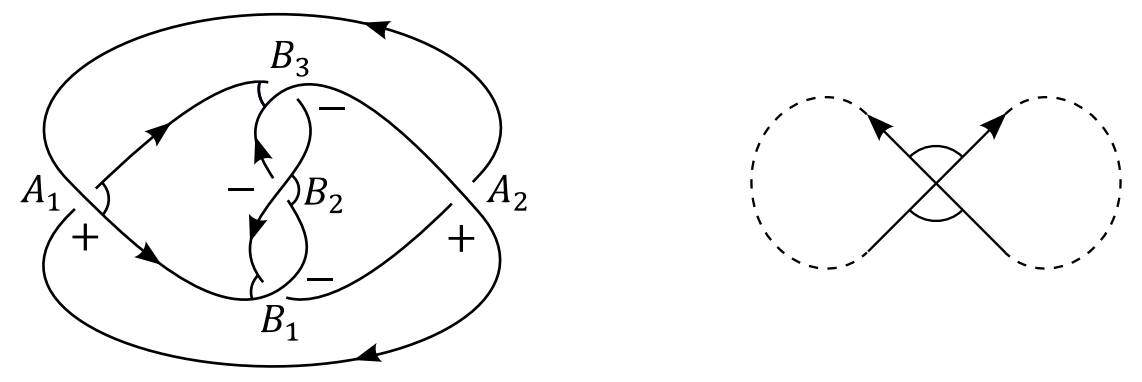

Лемма 1 (о направлениях кручений).

Для любого кручения в двух его вершинах либо оба направления ииклические, либо оба остовные.

Доказательство. Предположим, что направление кручения в одной из его вершин циклическое. Тогда концы диаграммы должны соединяться так, как указано на рисунке, и, очевидно, что направление кручения в другой его вершине также будет циклическим. Аналогично рассматривается случай остовного направления.

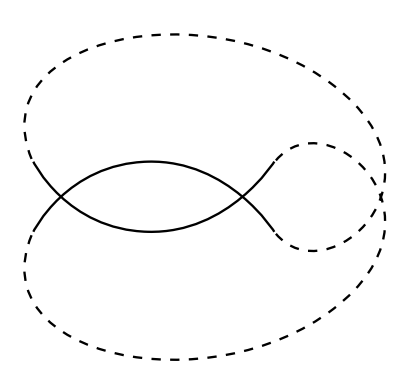

Кручение на плоской диаграмме называется ииклическим, если оба его направления циклические, и остовным, если оба направления остовные. 
Лемма 2 (об определении типа кручения). Кручение является остовным тогда и только тогда, когда при выборе некоторой ориентации плоской диаграммы ориентация сторон двуугольника, образующего кручение, задает одинаковую ориентачию окружности, образованной сторонами этого двуугольника.

Доказательство. Все очевидно следует из рисунка к лемме 1.

В любом направлении любой вершины плоской диаграммы $D_{K}$ может располагаться несколько кручений (см. пример 1). На множестве всех кручений, расположенных в данном направлении данной вершины, определим линейный порядок. Пусть на плоской диаграмме $D_{K}$ два кручения $A B_{1}$ и $A B_{2}$ имеют одинаковое направление в вершине $A$. Положим $A B_{1} \prec A B_{2}$, если $\alpha\left(A B_{1}\right) \subset \alpha\left(A B_{2}\right)$. Тогда $\beta_{A B_{1}} \supset \beta_{A B_{2}}$. На множестве всех кручений с данной вершиной $A$ определим теперь циклический порядок. Положим, что $A B_{1} \prec A B_{2} \prec A B_{3} \prec A B_{1}$, если кручения, расположенные в одном направлении, связаны определенным выше линейным порядком, а кручения, расположенные в разных направлениях, упорядочены в соответствии с естественным циклическим порядком направлений на плоской диаграмме $D_{K}$.

Пример 2. Для диаграммы из примера 1 имеем $A_{1} B_{1} \prec A_{1} B_{2} \prec A_{1} B_{3} \prec A_{1} B_{1}$.

Лемма 3. В любой вершине плоской диаграммы узла в каждом из двух ияклических направлений расположено хотя бы одно кручение, при этом возможно, что в обоих ииклических направлениях расположено одно и то же кручение, вершины которого совпадают.

Доказательство. Выберем некоторый перекресток $A$ на плоской диаграмме $D_{K}$ узла $K$ и обозначим через $\alpha$ и $\beta$ дуги, соединяющие концы перекрестка, как указано на рисунке. Если $\alpha$ и $\beta$ не пересекаются, то в вершине $A$ в обоих циклических направлениях, очевидно, расположено одно и то же кручение.
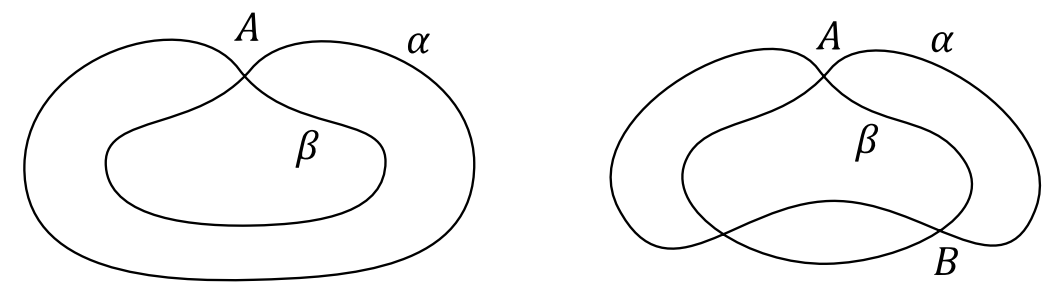

Если же $\alpha$ и $\beta$ пересекаются, то выберем перекресток $B$, ближайший к $A$ по дуге $\alpha$ с одного или другого циклического направления. Ясно, что тогда между $A$ и $B$ будет циклическое кручение.

3. Т-Графы. Т-Графом называется граф со следующей дополнительной структурой:

(1) каждая вершина снабжается знаком «плюс» или «минус»;

(2) для каждой вершины $A$ на множестве $E_{A}$ всех ребер, инцидентных вершине $A$, задан циклический порядок $\prec$, а также задано разбиение множества $E_{A}$ на 4 класса $E_{A}^{C 1}, E_{A}^{F 1}, E_{A}^{C 2}, E_{A}^{F 2}$, называемых направленияли ребер в вершине $A$, при этом классы $E_{A}^{C 1}$ и $E_{A}^{C 2}$, называемые ииклическими направлениями, непустые, и разбиение на классы согласовано с циклическим порядком $\prec$ в том смысле, что для любых ребер $l_{1} \in E_{A}^{C 1}, l_{2} \in E_{A}^{F 1}, l_{3} \in E_{A}^{C 2}$ и $l_{4} \in E_{A}^{F 2}$ имеем $l_{1} \prec l_{2} \prec l_{3} \prec l_{4} \prec l_{1}$; классы $E_{A}^{F 1}$ и $E_{A}^{F 2}$ будем называть остовными направлениями в вершине $A$;

(3) всякое ребро, инцидентное вершинам $A$ и $B$, либо принадлежит циклическим направлениям в обеих вершинах, либо остовным направлениям в обеих вершинах.

Закодируем структуру кручений на плоской диаграмме $D_{K}$ с помощью Т-графа, вершины которого соответствуют перекресткам на плоской диаграмме и снабжаются знаком «плюс» или «минус» в зависимости от типа перекрестка, а ребра Т-графа соответствуют кручениям на плоской диаграмме. Как было показано выше, в каждой вершине плоской диаграммы существуют 4 направления кручений, два из которых циклические и два остовные, в каждом из двух циклических направлений всякой вершины расположено хотя бы одно кручение, и у каждого кручения 
либо оба направления циклические, либо оба остовные, что полностью соответствует структуре Т-графа. Тем самым для любой плоской диаграммы узла Т-граф корректно определен.

Пример 3. Изобразим Т-граф для плоской диаграммы из примера 1, при этом циклические ребра будем изображать сплошными линиями, а остовные ребра - прерывистыми.

Циклическое и остовное направления в некоторой вершине Т-графа будем называть трансверсальными по отношению друг к другу.

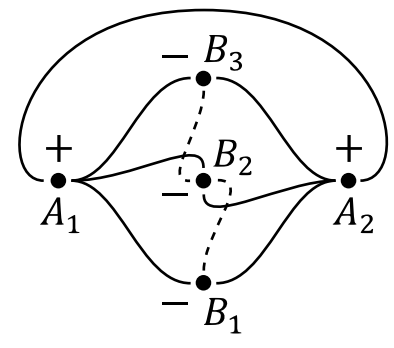

Теорема 1 (о свойствах Т-графа). Т-Граф любого узла $K$ обладает следующими свойствамu.

1. Для любых двух вершин $A$ и $B$ на Т-графе из вершины $A$ в данном направлении может выходить максимум одно ребро к вершине $B, u$, кроме того, $A$ и $B$ не могут быть соединены двумя ребрами в трансверсальных направлениях.

2. Если рассматривать плоскую диаграмму узла как планарный 4-валентный граф, то для любых двух вершин на этом графе, соединенных ребром, соответствуюшие вершины на Т-графе также соединены ребром.

Доказательство. Для заданной вершины $A$ на плоской диаграмме в заданном направлении, очевидно, существует максимум один двуугольник с непересекающимися сторонами и второй вершиной в заданной точке $B$. Кроме того, если вершины $A$ и $B$ на Т-графе соединены двумя ребрами в трансверсальных направлениях, то на плоской диаграмме эта ситуация будет выглядеть, как указано на рисунке, т.е. это будет диаграмма не узла, а двухкомпонентного зацепления. Тем самым свойство 1 доказано.

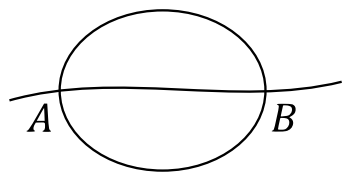

Пусть вершины $A$ и $B$ на плоской диаграмме, рассматриваемой как 4-валентный граф, соединены ребром $\gamma$. Обозначим через $\alpha_{1}$ и $\alpha_{2}$ дуги, выходящие из $A$ перпендикулярно $\gamma$, а через $\beta_{1}$ и $\beta_{2}$ дуги, выходящие из $B$ перпендикулярно $\gamma$. Ясно, что ровно одна из дуг $\alpha_{1}$ или $\alpha_{2}$ соединяется с одной из дуг $\beta_{1}$ или $\beta_{2}$, иначе получилась бы плоская диаграмма зацепления с более чем одной компонентой. Предположим, что $\alpha_{1}$ соединяется с $\beta_{1}$, тогда эта дуга не пересекается с $\gamma$ и образует кручение с вершинами $A$ и $B$, что доказывает свойство 2 .

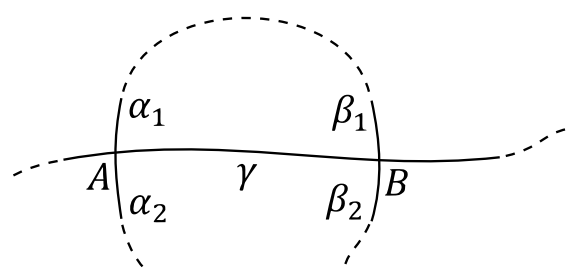

Путь в Т-графе будем называть ииклическим (остовным), если все составляющие его ребра циклические (остовные). Если два соседних ребра циклического либо остовного пути подходят к их общей вершине с противоположных направлений, то будем говорить, что путь является гладким в этой вершине. Если же ребра подходят к общей вершине с одного и того же направления, то будем говорить, что путь в этой вершине имеет касn.

Пример 4. На Т-графе из примера 3 путь $A_{1} B_{2} A_{2} B_{3} A_{1}$ является замкнутым циклическим путем, гладким в вершинах $B_{2}$ и $B_{3}$ и имеющим каспы в вершинах $A_{1}$ и $A_{2}$. 
Лемма 4. Для любого ииклического ребра $A B$ и любого остовного ребра $A C$ на T-графе, иниидентных общей вершине $A$, существует гладкий путь $\gamma$, соединяющий вершины $B$ и $C$, причем если путь $\gamma$ ииклический, то он подходит $\kappa$ вершине $B$ с того же направления, что и ребро $A B$, а если путь $\gamma$ остовный, то он подходит $к$ вершине $C$ с того же направления, что и ребро $A C$.

Доказательство. Пусть из вершины $A$ на Т-графе выходят одновременно циклическое ребро $A B$ и остовное ребро $A C$. Тогда на плоской диаграмме узла из вершины $A$ выходят циклическое и остовное кручения с общей дугой $\alpha$, и вершины $B$ и $C$ расположены на этой дуге. Пусть вершина $C$ расположена ближе к $A$ по дуге $\alpha$, чем $B$. Докажем, что тогда на Т-графе существует циклический путь $\gamma$ от $B$ к $C$, который подходит к $B$ с того же направления, что и ребро $A B$.

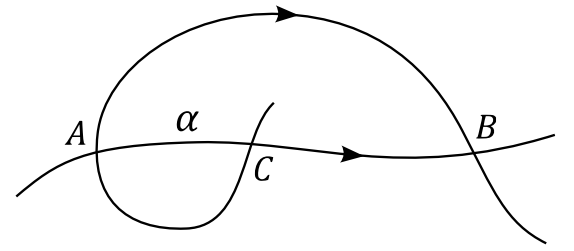

Обозначим через $A C$ сторону остовного кручения между $A$ и $C$, отличную от $\alpha$. Тогда если $A C$ и $B C$ не пересекаются, то между $B$ и $C$, очевидно, есть циклическое кручение (со сторонами $B C$ и $B A C)$, и искомый путь $\gamma$ состоит из одного этого кручения. Если же $A C$ и $B C$ пересекаются, то существование пути $\gamma$ легко доказывается индукцией по количеству точек пересечения. Обозначим через $D$ ближайшую к $C$ точку пересечения $A C$ и $B C$. Тогда, очевидно, между $D$ и $C$ расположено циклическое кручение, а между $A$ и $D$ расположено остовное кручение. Тем самым вместо точек $A, B$ и $C$ получаем аналогичную ситуацию для точек $A, B$ и $D$, но уже с меньшим числом пересечений дуги $A D$ (отличной от $\alpha$ ) и $B D$.

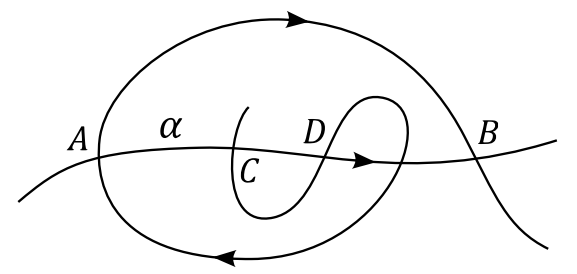

В полной аналогии проводится доказательство в случае, если вершина $B$ на плоской диаграмме расположена ближе к $A$ по дуге $\alpha$, чем $C$. Тогда, как несложно заметить, искомый путь $\gamma$ будет остовным.

Теорема 2 (о восстановлении узла по Т-графу). Т-граф однозначно определяет узел, т.е. не существует двух разных узлов с одинаковым Т-графом.

Доказательство. Известно, что плоская диаграмма узла полностью его определяет, поэтому достаточно показать, как по Т-графу восстановить плоскую диаграмму узла, которую можно представлять как 4-валентный граф с указанием структуры «проход-переход». Вершины Т-графа в точности соответствуют перекресткам плоской диаграммы, а знаки вершин Т-графа позволяют однозначно восстановить структуру «проход-переход» в каждом перекрестке. Осталось показать, как вершины соединяются между собой в 4-валентном графе, задающем плоскую диаграмму, т.е. для каждой вершины указать смежную с ней в каждом из 4 направлений.

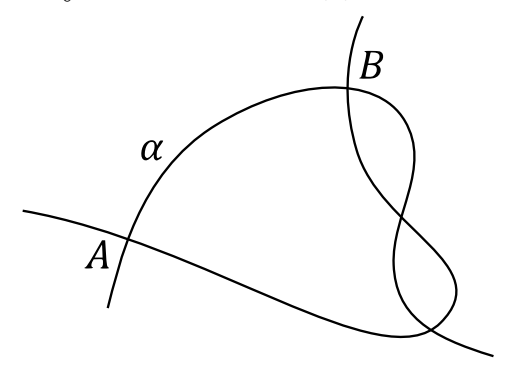


Рассмотрим плоскую диаграмму узла как 4-валентный граф, выберем некоторую вершину $A$ и одну из четырех дуг $\alpha$, выходящих из этой вершины. Обозначим через $B$ вершину, ближайшую к $A$ по дуге $\alpha$. С двух сторон от дуги $\alpha$ из вершины $A$ выходят два направления кручений: циклическое и остовное. По теореме 1 на Т-графе существует ребро, соединяющее $A$ и $B$. Этому ребру соответствует кручение на плоской диаграмме. Если это кручение циклическое и в остовном направлении кручений (а значит, и ребер на Т-графе) нет, то это позволяет по Т-графу восстановить соединение вершин $A$ и $B$ на 4-валентном графе, задающем плоскую диаграмму. Пусть на Т-графе существует циклическое ребро $A B$ и остовное ребро $A C$. Тогда по лемме 4 существует путь $\gamma$, соединяющий $B$ и $C$. Из доказательства леммы видим, что если путь $\gamma$ циклический, то ближайшей к $A$ вершиной по дуге $\alpha$ будет $C$, а если путь $\gamma$ остовный, то ближайшей будет $B$. $\square$

Рассмотрим два преобразования Т-графов (на рисунках также указано соответствующее преобразование фрагмента плоской диаграммы):

(i) редуцирование гладкого пути:
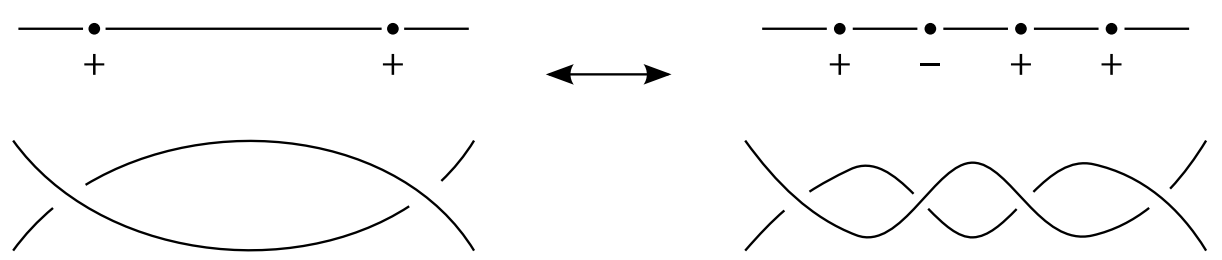

(ii) редуцирование развилок:
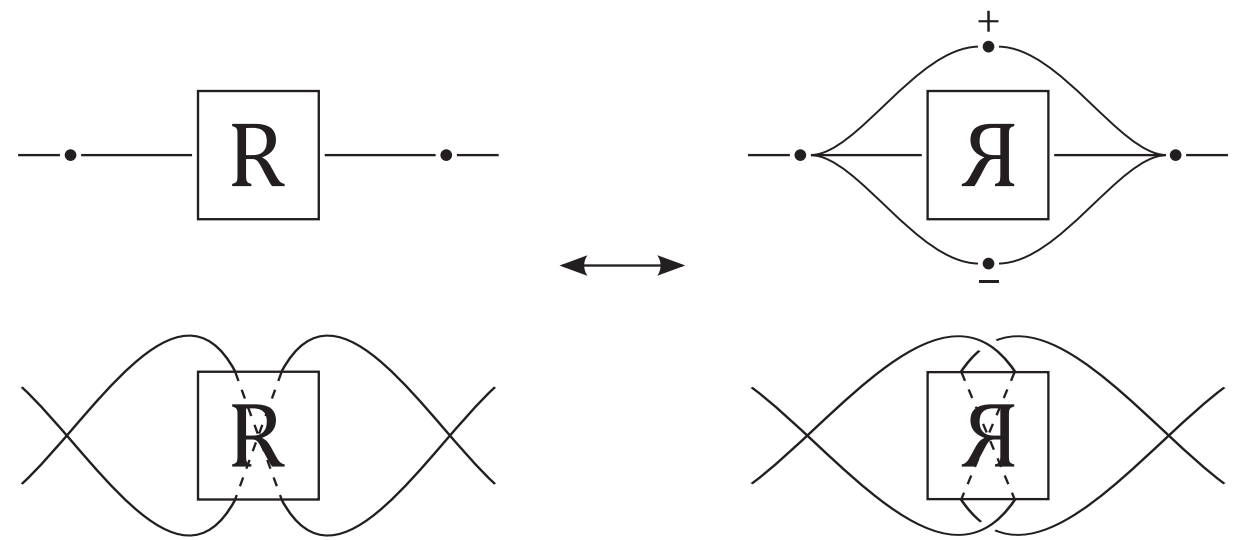

Здесь буквой $R$ обозначен фрагмент Т-графа и плоской диаграммы. В процессе преобразования этот фрагмент отражается относительно своей вертикальной оси.

Данные преобразования, очевидно, позволяют перейти к другому Т-графу того же самого узла.

4. Пример распознавания тривиального узла. В силу теоремы 2 Т-граф есть не просто способ кодирования кручений на плоской диаграмме узла, а фактически способ кодирования самого узла. Кодирование узлов с помощью Т-графов позволяет в некоторых случаях проще решать задачу распознавания узлов. Приведем характерный пример.

Пример 5. Рассмотрим плоскую диаграмму тривиального узла и соответствующий этой диаграмме Т-граф:
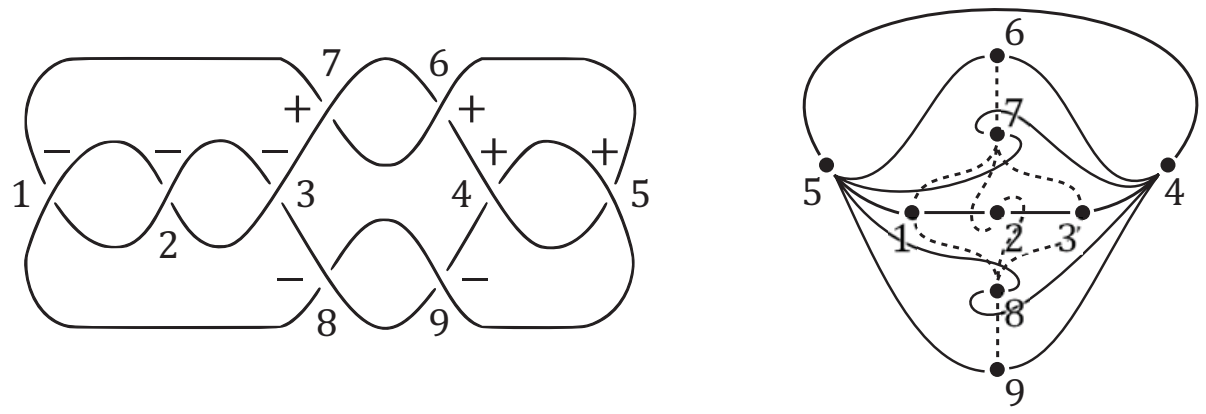
Известно (см. [2]), что данную плоскую диаграмму нельзя преобразовать в тривиальную диаграмму (не имеющую перекрестков) последовательным применением движений Рейдемейстера так, чтобы на каждом шаге количество перекрестков не увеличивалось. В то же время построенный по этой диаграмме Т-граф легко преобразуется в тривиальный без увеличения его сложности (количества вершин и количества ребер) на каждом шаге, что позволяет легко распознать тривиальность узла. В самом деле, применим к Т-графу редуцирование развилок, сократив вершины 6 и 9, имеющие противоположные знаки, а потом точно так же сократив вершины 7 и 8.

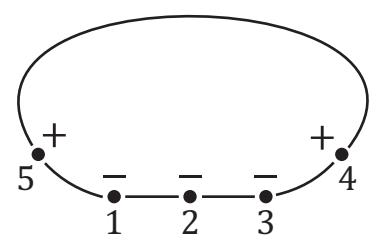

Далее применим к полученному Т-графу редуцирование гладкого пути, сократив вершины 1 и 5 , имеющие противоположные знаки, а потом сократив вершины 3 и 4 . Получим Т-граф с одной вершиной 2, которая, очевидно, задает тривиальный узел (его плоская диаграмма изображена рядом).
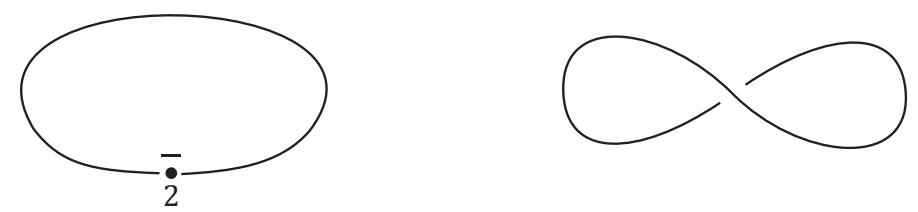

\section{СПИСОК ЛИТЕРАТУРЫ}

1. Дынников И. А. Алгоритмы распознавания в теории узлов// Усп. мат. наук. -2003 . -58 , № 6 (354). - C. 45-92.

2. Goeritz L. Bemerkungen zur Knotentheorie// Abh. Math. Sem. Univ. Hamburg. — 1934. — 10. — P. 201210 .

Бирюков Олег Николаевич

Московский государственный технический университет им. Н. Э. Баумана

E-mail: onbiryukov@yandex.ru 